\title{
Internal consistency: Reports of its death are premature
}

\author{
Ronald R. Holden • Ira H. Bernstein
}

Published online: 1 February 2013

(C) Psychonomic Society, Inc. 2013

\begin{abstract}
McCrae, Kurtz, Yamagata, and Terracciano (Personality and Social Psychology Review 15:28-50, 2011) presented data supporting the position that test-retest reliability is more important for methods of personality assessment than is internal-consistency reliability. We raise two points not adequately considered in their findings. First, not all personality assessment is concerned with assessing traits, for which temporal stability is clearly important. Much research (e.g., therapy outcome studies) is concerned with assessing states, in which an emphasis on temporal stability can lead to the underestimation of change. Second, we suggest that using the literature as a basis for their position causes the role of internal consistency to be underestimated. This is because manuscripts are frequently rejected because their measures possess low internal consistency, but are rarely rejected because of low temporal stability. Thus, the effects of internal consistency are range-restricted to an unknown amount, because investigators do not have access to studies that have been rejected due to low internal consistency.
\end{abstract}

Keywords Internal consistency · Test construction methods · Personality assessment

McCrae et al. (2011) was published in Personality and Social Psychology Review. which does not provide an opportunity for subsequent commentary. Given the methodological implications of McCrae et al.'s article, Behavior Research Methods was regarded as an appropriate forum.

R. R. Holden $(\bowtie)$

Department of Psychology, Queen's University,

Kingston, ON K7L 3N6, Canada

e-mail: holdenr@queensu.ca

I. H. Bernstein

Department of Clinical Sciences, University of Texas Health

Science Center at Dallas, Dallas, TX, USA
McCrae, Kurtz, Yamagata, and Terracciano (2011) provided important insights into the relevance of test-retest reliability for personality trait scale validity, and in doing so challenged longstanding beliefs regarding the role of internal consistency for personality assessment. In collating and filtering through a multitude of research studies and data archives, the authors evaluated the individual and relative merits of different forms of reliability for statistically predicting various operationalizations of scale validity/goodness/quality. Overall, their article's empirical findings support the statistical importance of test-retest reliability and seem to find, in stark contrast to traditional psychometric belief, little value or added value for considering internal-consistency reliability (e.g., coefficient alpha). Although the article's focus was primarily on the facet and domain scales of the Revised NEO Personality Inventory (Costa \& McCrae, 1992), supplementary evidence was also provided for other measures, and the authors implied that their findings may transcend specific inventories and be generally applicable to personality scales. The authors summarized by advocating testretest reliability and indicating the "limited utility" (p. 28) associated with internal-consistency reliability.

Although we appreciate the many positive features of the McCrae et al. (2011) contribution, we strongly feel that it is essential to recognize the potential limitations associated with their findings. These limitations include their focus on already developed scales and their use of select measures of scale quality. Perhaps more important, however, are two additional, particularly noteworthy limitations: the consideration only of the measurement of trait-like personality characteristics, and the fact that articles in the literature are range-restricted with regard to internal consistency, because that is a major feature of their evaluation - which is, perhaps ironically, closely related to McCrae et al.'s thesis. The range restriction arises from the fact that journal reviewers are trained to place heavy emphasis upon this test characteristic. This is not surprising, given that current texts on psychometrics 
often summarize chapters on reliability by indicating that internal-consistency reliability is "the preferred reliability estimate” (Reynolds \& Livingston, 2012, p. 125).

In examining associations between reliability and scale quality, McCrae et al. (2011) focused on finished, published scales, and consequently did not fully address various stages of scale development. As such, aspects of test construction related to construct specification, item pool generation, and item selection were not included. Endorsed methods for test construction (American Educational Research Association, American Psychological Association, \& National Council on Measurement in Education, 1999) involve construct measurement, in which, typically, a characteristic of interest is defined; on the basis of this definition, a table of specifications is outlined; items are written to represent elements in the table of specifications; and items are then empirically evaluated and selected on the basis of their properties (usually using item total correlations or item factor loadings, from which content homogeneity subsequently emerges; or, alternatively, using a contrasted-groups approach in which item-discriminating power is targeted). The use of a test-retest method for scale development because of the "limited utility" (McCrae et al., 2011, p. 28) of internal-consistency methods, although intriguing, seems premature without more empirical research into the item metrics of scale development (see also Watson, 2004). As was noted by McCrae et al., past research (Ashton \& Goldberg, 1973; Jackson, 1975) has demonstrated the utility of the internal-consistency approach over an external-empirical strategy to test construction, but scant work has been done on the use of item test-retest reliability as a basis for item selection in scale development. Nevo (1977) has compared item internal consistency and item test-retest stability selection procedures, but not with regard to item validity or quality. Furthermore, he noted issues of practicality, whereby a test-retest item selection procedure necessitates double-testing of respondents over a yet-to-be-determined optimal time interval. Thus, whereas the utility of internal-consistency approaches to scale development has been established, the use of a testretest method for item selection and scale development is uncharted territory and cannot yet be recommended.

In evaluating scale quality, McCrae et al. (2011) operationalized "goodness" using three indices: longitudinal stability, heritability, and cross-observer agreement. Although these criteria are reasonable, practically available for their research purposes, and not inappropriate, the indices are a select, incomplete set. Consider that, despite acknowledging the operational linkage between the criterion of longitudinal stability and the predictor of retest reliability, McCrae et al. opted to use this outcome, which is biased toward favoring retest reliability over other, non-spuriously-inflated predictors. Furthermore, their choice of heritability as an outcome is grounded in a specific evolutionary perspective and is a criterion that is not standardly employed in scale validation or item selection. Finally, issues of predictive validity (i.e., predicting relevant outcomes) are not included in the McCrae et al. criteria. This is a major omission. Much of the resurgence of personality assessment and of the popularity of Big Five measures of personality has occurred because of the ability of personality dimensions to predict job performance criteria (see Barrack \& Mount, 1991). In providing validity evidence based on relations to other variables, "Measures other than test scores, such as performance criteria, are often used in employment settings" (American Educational Research Association et al., 1999, p. 13). As such, predictive validity for relevant outcomes is a highly relevant and important practical criterion that is not addressed by McCrae et al.

We now make two primary arguments in this article. The first is that McCrae et al. (2011) underemphasized an important aspect of personality research, the assessment of personality change. In that context, measures with too great a temporal stability will underestimate the change that occurs in situations such as psychotherapy. Our second argument is statistical in nature: Constructing reliable measures has been a major factor in personality research throughout much of its history (cf. Nunnally \& Bernstein, 1994, and its preceding editions). Because of this, measures with low internal consistency have a difficult time passing peer review. The result is that the published literature rangerestricts this variable so that coefficients alpha and related statistics are inclined to correlate poorly with measures such as those that McCrae et al. studied.

\section{Trait versus state measures}

The emphasis of the McCrae et al. (2011) argument is on stable personality differences (i.e., traits). Although personality assessment usually focuses on the measurement of traits, many other individual differences of psychological interest of course are transient or temporary. Emotions, states, and moods represent a few domains in which the underlying constructs are theorized to exist but are postulated to be of quite limited duration. Consider also circumstances in which there is a desire to construct an instrument to measure change (e.g., when evaluating a pharmacological intervention for the treatment of depression or examining the merits of relaxation therapy for lowering high blood pressure). In such circumstances, the effective evaluation of treatments requires the use of a construct-valid measure capable of detecting differences from a baseline. An overemphasis on the stability of measures can run contrary to demonstrating the existence of therapeutic change. As an extreme example, consider an item such as "I have been arrested for shoplifting" on a scale of social deviance that is being used to evaluate the effectiveness of an offender rehabilitation program. Once it is true for an individual, 
logically, this item must always be true. As such, the item likely manifests extremely high test-retest reliability, and yet it clearly and inappropriately mitigates demonstrating the effectiveness of even the most efficacious rehabilitation program. Thus, methods that emphasize test-retest stability are associated with limitations, just as potential limitations are also associated with extremely high internal consistencies. Where the boundaries for test-retest reliabilities are has yet to be delineated.

\section{Range restriction of measures based on low internal consistency}

In examining already-existing scales, and in particular, NEO domain and facet scales, McCrae et al. (2011) limited the values associated with internal-consistency reliability. Their Table 2 (p. 32) indicates median NEO scale-internal consistencies of .71 (test manual sample), .76 (college sample), or .73 (research sample). This is not surprising, and it attests to the care and success with which the NEO inventories were developed. It may also indicate a publication bias against research in which low internal-consistency reliabilities are found. The wealth of data analyzed by the authors further indicates the durability and popularity of this elite set of personality scales. However, the very psychometric strengths of the NEO scales present an important and well-known statistical issue for examining how scale properties relate to scale quality-restriction of range. Because the NEO scales were successfully constructed to foster scale homogeneity, the range along that characteristic (theoretically 0.00 to 1.00 ) is sampled neither randomly nor representatively, but only very selectively and representative of only a narrow band. Consequently, the subsequent relationships of other variables with indices of internal consistency will be underestimated and not reflective of the true associations.

We emphasize that our criticism is not directed toward the NEO scales, but to the general hypothesis that temporal stability is more important than internal consistency. As a reviewer noted, the corresponding ranges of the temporalstability measure in the three samples cited above are .25 $(.56-.81), .37(.50-.87)$, and $.32(.50-.82)$, whereas the ranges of internal-consistency estimates (from one different sample and the second of the samples cited above) are .19 (.67-.86) and .21 (.70-.91). By one definition, range is more restricted for the temporal-stability measures than for internal consistency in these specific studies. However, the data in this table came from well-established scales rather than from those seeking to gain recognition through publication in a major journal. Consequently, these data do not represent the reliability estimates obtained for tests in general, which may be unknowable if the article is not published. Likewise, they represent data on a highly evolved set of measures. Many other measures reported in the literature are basically in their infancy.

A temporal-stability estimate is of course a joint property of the sample and test, and not just of the test itself. Another way to describe this is to say that a reliability estimate is a property of the obtained scores in a given sample rather than of the test per se. As another reviewer noted, reliability generalization studies typically deal with the issue of how such measures actually do vary across samples (see Henson \& Thompson, 2002; Vacha-Haase, Henson, \& Caruso, 2002).

With an emphasis on internal consistency in scale construction resulting in psychometrically elite scales that, because of their development, have a restricted range of internal consistency, we can conjecture an alternative, hypothetical history and outcome. Of course, we cannot provide empirical support for this position, because the hypothetical nature of these studies does not make them available for citation.

Suppose, in the 1980s, that test developers constructed a personality inventory using item selection methods that focused on item test-retest correlations rather than on item total correlations or item factor loadings. Furthermore, suppose that the inventory was quite successful, widely used, and had large data archives accumulated for it. After 30 years, investigators decided to undertake analyses identical to those of McCrae et al. (2011). We suggest that, for this hypothetical alternative, the findings would be opposite those of McCrae et al.; because, in actuality, the NEO scale development emphasized factor-analytic structure and internal consistency, it is far from surprising that systematic variations in scale properties would be attributable to something other than internal consistency. For our hypothetical, alternative inventory, developed to optimize temporal stability, systematic differences in scale quality would be attributable to factors other than stability over time. This is because item selection on the basis of a particular property produces a restriction of variability in that property and in the associations of that property with other characteristics.

In highlighting some limitations of McCrae et al. (2011), we do not dispute their empirical findings but, rather, take issue with the perspective of a much-diminished role for internalconsistency reliability. Although internal-consistency reliability can be overemphasized, such as when fidelity compromises bandwidth and content validity (e.g., a 16-item scale consisting of one item copied 15 times), we continue to advocate the methods of the Standards for Educational and Psychological Testing (American Educational Research Association et al., 1999), whereby, in many instances, "an internal consistency coefficient may be preferred," and "It should not be inferred that alternate-form or test-retest coefficients ... are always preferable to internal consistency coefficients" (p. 28). 


\section{Conclusion}

Although we acknowledge the importance of temporal stability in studies of trait measures and applaud McCrae et al.'s (2011) forcefulness, we feel that they have gone too far in ignoring the importance of measuring state-like measures for which too much temporal stability is a liability, and that they have neglected an important statistical artifact underlying their failure to find a relationship between internal consistency and personality scale validity.

Author note This research was supported by the Social Sciences and Humanities Research Council of Canada.

\section{References}

American Educational Research Association, American Psychological Association, \& National Council on Measurement in Education. (1999). Standards for educational and psychological testing. Washington, DC: American Educational Research Association.

Ashton, S. G., \& Goldberg, L. R. (1973). In response to Jackson's challenge: The comparative validity of personality scales constructed by the external (empirical) strategy and scales developed intuitively by experts, novices, and laymen. Journal of Research in Personality, 7, 1-20.
Barrack, M. R., \& Mount, M. K. (1991). The Big Five personality dimensions and job performance: A meta-analysis. Personnel Psychology, 44, 1-26.

Costa, P. T., Jr., \& McCrae, R. R. (1992). Revised NEO Personality Inventory (NEO PI-R) and NEO Five-Factor Inventory (NEO-FFI) professional manual. Odessa: Psychological Assessment Resources.

Henson, R. K., \& Thompson, B. (2002). Characterizing measurement error in scores across studies: Some recommendations for conducting "reliability generalization" studies. Measurement and Evaluation in Counseling and Development, 35, 113-127.

Jackson, D. N. (1975). The relative validity of scales prepared by naïve item writers and those based on empirical methods of personality scale construction. Educational and Psychological Measurement, $35,361-370$.

McCrae, R. R., Kurtz, J. E., Yamagata, S., \& Terracciano, A. (2011). Internal consistency, retest reliability, and their implications for personality scale validity. Personality and Social Psychology Review, 15, 28-50.

Nevo, B. (1977). Using item test-retest stability (ITRS) as a criterion for item selection: An empirical study. Educational and Psychological Measurement, 37, 847-852.

Nunnally, J. C., \& Bernstein, I. H. (1994). Psychometric theory (3rd ed.). New York: McGraw-Hill.

Reynolds, C. R., \& Livingston, R. B. (2012). Mastering modern psychological testing: Theory \& methods. New York: Pearson.

Vacha-Haase, T., Henson, R. K., \& Caruso, J. (2002). Reliability generalization: Moving toward improved understanding and use of score reliability. Educational and Psychological Measurement, $62,562-569$.

Watson, D. (2004). Stability versus change, dependability versus error: Issues in the assessment of personality over time. Journal of Research in Personality, 38, 319-350. 